\title{
Avatars in space
}

\section{Why bother to colonize space when electronic devices do it so much better?}

\section{Geoffrey A. Landis}

he twenty-first century completed the era when biological species expanded (briefly) their range of habitation into space. Space is a harsh environment for fragile biological machines, which must stay inside carefully climate-controlled cans of air. As we approach the end of the third millennium, our electronic descendants have no such problem. After all, robots are stronger and tougher, don't need lifesupport systems, and don't get bored. They think of space as home.

Our electronic avatars that thrive in space are about the size of a child's fist (many of them are considerably smaller), and look more like a spider than a spacecraft. Thanks to our avatars, we have spread out across all the planets of our Solar System and many others, and the dark spaces in between as well - but there aren't any humans in space at all, only our electronic ghosts, smarter and faster, engineered for the harsh conditions of space. And maybe every now and then, they think about us biological humans a little, and sometimes even reminisce about living on a planet.

On the surface of the Earth, the superfast, microscopic computers have their applications, too. No task that requires intelligence is left to biological humans. With computer memories that can store information at a density of several bits per atom, an entire biological human's neural state can be encoded in just a few exabytes - less than a milligram of material. When you can scan your entire brain, download it to a chip, and have the chip interact for you inside the virtual worlds of the worldwide interconnected computer network - and then download itself back to you - some people have problems remembering which one 'they' really are. And so humans went into space after all, using high-bandwidth laser links to upload their neural software to space computers.

The space robots, however, continued to evolve and eventually speciate. Some, based on high-temperature silicon-carbide and diamond-based semiconductor materials, moved inward towards Mercury. With copious energy in the form of sunlight, and with resources to be mined from the crust and the polar caps, Mercury seemed the perfect place for machine colonies.

Other machines diverged away from semiconductors, and began to use Josephson junctions for computation. These superconducting machines moved away from the Sun, toward the outermost gas giants and the
Kuiper belt. With superconducting power buses and Josephson electronics, they didn't really need much power.

The main belt asteroids, in between the two realms, served as raw materials for both. The divergence of electronic species led to inevitable conflict over resources that were valuable to both. Even now, wars are being fought between the machines over resources in this middle area; no doubt fiercer wars are yet to come, as ever larger and more ambitious projects make resources more valuable.

The planets themselves - outside Mercury - are of only minor interest to our electronic avatars. With too much free oxygen and liquid water, and unreliable sunlight, Earth is a poor place for machines.

The avatars have their own society. With several trillion individuals, their society is so complicated that it will never be possible for a biological human to understand it. Their art, their music, their intricate social structure - the humans that have uploaded into space are only a negligibly small part of it.

Far below, and almost ignored by the enormous electronic ecosystem in space, biological species still thrive on Earth. No longer on the cutting edge of science - every problem that could possibly be understood by a biological mind has long ago been studied to completion by silicon-carbide brains - the humans devote themselves to the art of living graciously, in harmony with an ecosystem that is now understood in exhaustive detail. Art, history, literature, sports, recreation, religion, philosophy: humans still have plenty of activities to which they can devote themselves. Work and war are equally forgotten what would be the point? Those who want territory, or resources, or power, eventually decide to upload themselves into space.

As the millennium draws to a close, the Josephson computers are now reaching for the stars the slow way, colonizing each one of ten trillion comets in the dark spaces between the stars. The Mercury machines, on the other hand, are reaching for the stars in the fast lane: they have constructed an enormous particle-beam accelerator, closer to the Sun than Mercury. Machines no larger than a grain of rice are sailing on particle beams to 500 of the nearest stars, at $89 \%$ of the speed of light. These machines are only there to fly past and report, but if they find conditions hospitable - and for machines that have no need for planets, the range of 'hospitable' is very wide indeed - the next generation will be colonists.

The future looks interesting.

Geoffrey A. Landis was a member of the Mars Pathfinder science team, and is currently working on hardware for future Mars missions. His first novel, Mars Crossing, will appear in late 2000. http://www.sff.net/people/geoffrey.landis

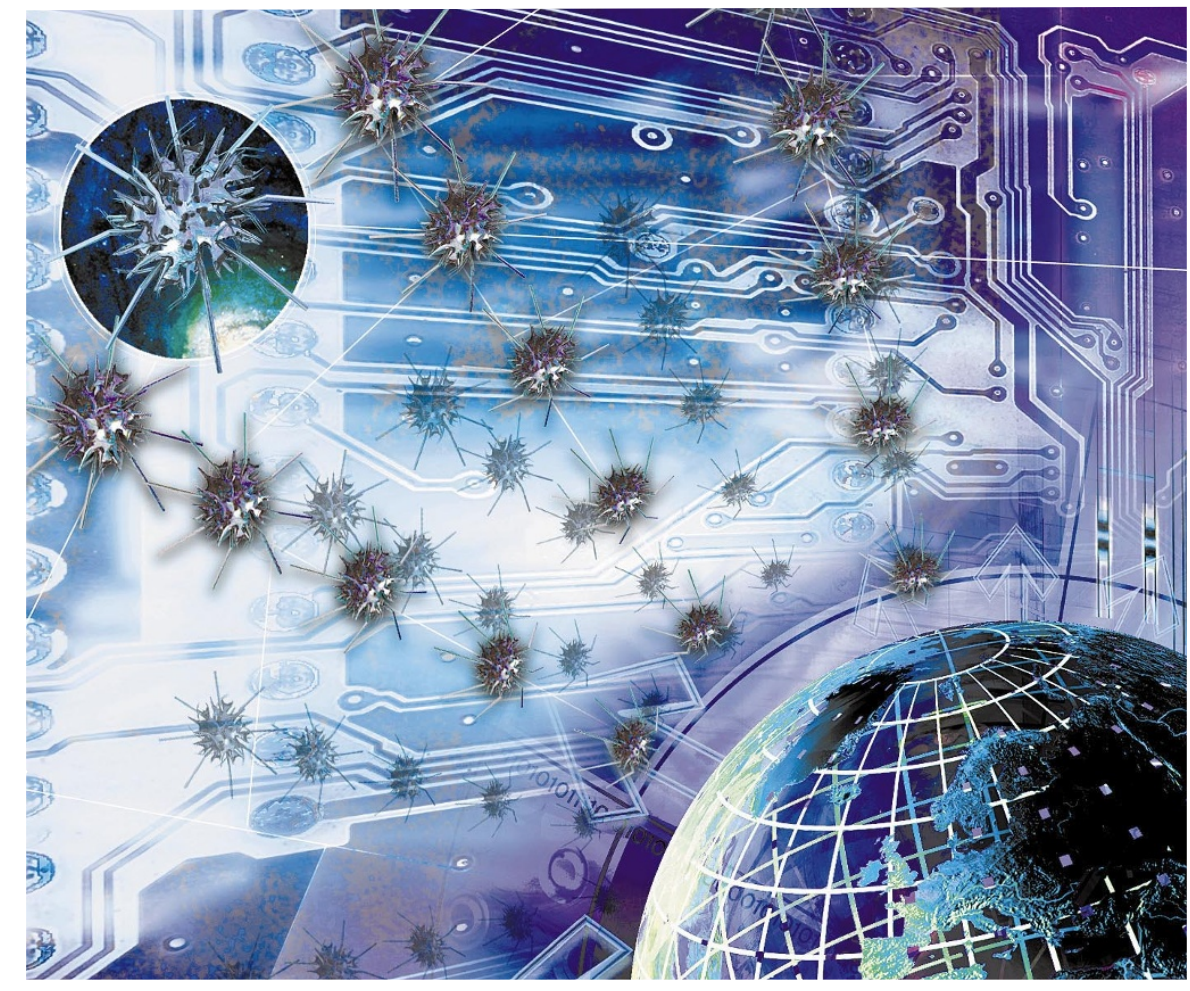

